\title{
ON GLOBAL TRAJECTORY TRACKING CONTROL OF ROBOT MANIPULATORS WITH A DELAYED FEEDBACK
}

\author{
Aleksandr Andreev \\ Department of ISCT \\ Ulyanovsk State University \\ Russia \\ asa5208@mail.ru
}

\author{
Olga Peregudova \\ Department of ISCT \\ Ulyanovsk State University \\ Russia \\ peregudovaoa@gmail.com
}

Article history:

Received 27.10.2021, Accepted 25.11.2021

\begin{abstract}
In this paper, the trajectory tracking control problem of a robot manipulator with cylindrical joints is considered by means of a nonlinear PD controller taking into account the delayed feedback structure. The conclusion about stability of a closed-loop system is obtained on the basis of the development of the direct Lyapunov method in the study of the stability property for a non-autonomous functional differential equation by constructing a Lyapunov functional with a semi-definite time derivative.
\end{abstract}

\section{Key words}

Robots manipulators, Motion Control Systems, Trajectory Tracking, Systems with time-delays, Stability of nonlinear systems, Lyapunov methods.

\section{Introduction}

Among the main control problems for robotic systems is the trajectory tracking of manipulators, mobile robots, mobile manipulators, unmanned aerial vehicles, and other systems. Proportional-differentiating (PD) controllers are widely used in solving this problem. A fairly complete current state of research on the use of PD controllers in the control of manipulators including mobile ones is available in the papers [Aguinaga-Ruiz et al., 2009], [Kim et al., 2017], [Moreno-Valenzuela et al., 2010], [Nunes et al., 2008], [Oliveira et al., 2015], [Rigatos, 2020]. At the same time, certain difficulties lie in the need to compensate for centrifugal, Coriolis and gravitational forces. In robotics, such a drawback can be avoided by using relay controllers [Khalil, 2001], [Spong et al., 2004], [Utkin et al., 2020]. Besides, such controllers are simple to implement, and they have the ability to achieve a goal in a finite time, etc. But in practice, relay control laws reveal some disadvantages which include: the need for complete control, significant energy losses due to the use of maximum modulus of control actions, and high-frequency oscillations of the state vector of the system in a sliding mode leading to unwanted vibrations of the mechanical elements of the control structure. Accordingly, the problem of using relay controllers remains relevant. A sufficiently detailed analysis of the works on tracking feasible trajectories in underactuated manipulators with an interesting solution to the control problem of a two-link manipulator is available in the paper [Penaloza-Mejia et al., 2013].

A significant fleet of robotic manipulators consists of manipulators with cylindrical joints. The dynamics of such manipulators is described by angular coordinates. In this case, the motion equations are described by periodic functions in these variables. This specificity was taken into account in [Andreev and Peregudova, 2020] by constructing a nonlinear PD controller in a cylindrical phase space. A definite advantage of such regulators is the reduction in energy consumption for control.

Modeling a number of engineering systems with many inputs leads to the need to take into account the delay of input signals for the developed models. These include, for example, multi-agent systems, chemical processes, rolling mills, remotely controlled robotic systems, etc. [Fischer et al., 2013], [Bagheri et al., 2019], [BekiarisLiberis and Krstic, 2017].

The paper [Bekiaris-Liberis and Krstic, 2017] presents a methodology for constructing the predictor-feedback control in the stabilization problem of nonlinear systems with delay. Global asymptotic stability is proved both by constructing the Lyapunov functional and by estimating the solutions of the system.

The paper [Bagheri et al., 2019] investigates the control problem of a manipulator with high performance 
based on a predictor approach which makes it possible to compensate a constant input lag. The controller was tested on the 7-DOF (degrees of freedom) manipulator. Predictor-based control effectively compensated the input delays and ensured the stability of the closed-loop system.

The paper [Castaneda et al., 2018] deals with the control of a robotic system with a constant delay of input variables.The system is modeled as a linear perturbed system in which the lumped input variables are estimated and then compensated for by extended state observer controller. The efficiency of the solution is shown using a two DOF robotic manipulator.

The most convenient method for finding the admissible delay in the corresponding problems is the direct Lyapunov method in studying the stability of functional differential equations [Hale, 1977].

The aim of this paper is to study, in a nonlinear formulation, the trajectory tracking control problem of a serial robot manipulator with cylindrical joints with the determination of the admissible delay in the feedback structure. To solve it, the direct Lyapunov method is developed in the direction of determining the properties of asymptotic stability, attraction of solutions of a nonautonomous functional differential equation with a righthand side which is periodic in a part of independent variables.

\section{Preliminaries}

Let $\mathrm{R}^{p}$ be a linear real space of $p$-vectors $x$ with some norm $|x|$; let $h_{0}>0$ be some real; let $\mathrm{C}$ be the Banach space of continuous functions $\varphi:\left[-h_{0}, 0\right] \rightarrow \mathrm{R}^{p}$ with the norm $\|\varphi\|=\max \left(|\varphi(s)|,-h_{0} \leq s \leq 0\right)$. For each positive real $H, 0<H<\infty$ denote by $\mathrm{C}_{H}$ the subset of $\mathrm{C}$ as follows $\mathrm{C}_{H}=\{\varphi \in \mathrm{C}:\|\varphi\| \leq H\}$. For each continuous function $x: \mathrm{R} \rightarrow \mathrm{R}^{p}$ and each $t \in \mathrm{R}^{+}$, the function $x_{t} \in \mathrm{C}$ is defined by the equality $x_{t}(s)=x(t+s),-h_{0} \leq s \leq 0$.

Consider a non-autonomous functional differential equation

$$
\dot{x}(t)=f\left(t, x_{t}\right),
$$

where $\dot{x}(t)$ is an upper right-hand derivative, $f: \mathrm{R} \times$ $\mathrm{C} \rightarrow \mathrm{R}^{p}$ is a continuous function satisfying the conditions

$$
\begin{array}{r}
|f(t, \varphi)| \leq m(H), \\
\left|f\left(t_{2}, \varphi^{(2)}\right)-f\left(t_{1}, \varphi^{(1)}\right)\right| \leq \\
L(H)\left(\left|t_{2}-t_{1}\right|+\right. \\
\left.+|| \varphi^{(2)}-\varphi^{(1)} \|\right) \\
\forall(t, \varphi),\left(t_{1}, \varphi^{(1)}\right),\left(t_{2}, \varphi^{(2)}\right) \in \mathrm{R} \times \mathrm{C}_{H}
\end{array}
$$

Under the conditions (2), for each initial data $(\alpha, \varphi) \in$ $\mathrm{R} \times \mathrm{C}_{H}$ there exists a unique solution $x=x(t, \alpha, \varphi)$ of (1), $x_{t}(\alpha, \varphi)=\varphi$ defined on the interval $\left[\alpha-h_{0}, \beta\right)$, $\beta>\alpha$, and the following holds $\left\|x_{t}(\alpha, \varphi)\right\| \rightarrow \infty$ as $t \rightarrow \beta$, if $\beta<\infty$.
Let $r_{n}$ be some monotonically increasing sequence, $0<r_{1}<r_{2}<\ldots<r_{n}<\ldots, r_{n} \rightarrow \infty$ as $n \rightarrow \infty$.

For each number $r_{n}$ denote by $K_{n} \subset \mathrm{C}$ the set of functions $\varphi \in \mathrm{C}$ such as

$$
|\varphi(s)| \leq r_{n}, \quad\left|\varphi\left(s_{2}\right)-\varphi\left(s_{1}\right)\right| \leq m\left(r_{n}\right)\left|s_{2}-s_{1}\right|
$$

for all $s, s_{1}, s_{2} \in\left[-h_{0}, 0\right]$.

Note that the sets $K_{n}(n=1,2,3, \ldots)$ are compact. Assume that

$$
D=\bigcup_{n=1}^{\infty} K_{n} .
$$

Claim 2.1. If $x=x(t, \alpha, \varphi)$ is a solution of (1) defined on the interval $\left[\alpha-h_{0}, \infty\right)$, then $x_{t}(\alpha, \varphi) \in D$ for all $t \in\left[\alpha+h_{0}, \infty\right)$. In particular, if $\varphi \in D$, then $x_{t}(\alpha, \varphi) \in$ $D$ for all $t \in[\alpha, \beta)$.

Let $F$ be the set of all continuous functions $f: \mathrm{R} \times$ $\Gamma \rightarrow \mathrm{R}^{p}$. Define the convergence in $F$ according to a compact-open topology [Andreev, 2009]; namely, the sequence $\left\{f_{n} \in F\right\}$ converges to $f \in F$, if for each compact set $K \subset R \times \Gamma$ and for each $\varepsilon>0$ the following estimate holds $\left|f_{n}(t, \varphi)-f(t, \varphi)\right|<\varepsilon$ for all $n \geq N(\varepsilon)$ and $(t, \varphi) \in K$. This convergence is metrizable.

Claim 2.2. Let the function $f: R \times \Gamma \rightarrow \mathrm{R}^{p}$ satisfy the conditions like (2). Then, the family of translates $\left\{f_{\tau}(t, \varphi)=f(\tau+t, \varphi), \tau \in \mathrm{R}^{+}\right\}$of the function $f$ is precompact in $F$.

Definition 2.1. For the right-hand side $f$ of (1) denote by $G(f)$ the closure of the set $\left\{f_{\tau}(t, \varphi)\right\}$. The set of the equations

$$
\dot{x}(t)=f^{*}\left(t, x_{t}\right), \quad f^{*} \in G(f)
$$

forms a family of limiting equations for (1) [Andreev, 2009].

Note that the functions $f^{*} \in G(f)$ satisfy the conditions (2). Therefore, the solution $x=x^{*}(t, 0, \varphi)$ of each equation (3) for each point $\varphi \in C$ is unique.

Definition 2.2. Let $x=x(t, \alpha, \varphi)$ be a solution of (1) defined for all $t \geq \alpha-h_{0}$. The set

$$
\begin{array}{r}
\omega^{+}(x(t, \alpha, \varphi))=\left\{q \in \mathrm{R}^{p}: \exists t_{n} \rightarrow \infty,\right. \\
\left.x\left(t_{n}, \alpha, \varphi\right) \rightarrow q \text { as } n \rightarrow \infty\right\}
\end{array}
$$

is called a positive limit set of $x(t, \alpha, \varphi)$ in $R^{p}$.

Definition 2.3. The set $M \subset \mathrm{R}^{p}$ is called quasiinvariant with respect to the family of limiting equations (3), if for each point $q \in M$ there exists an equation of the family (3) such that for the solution $x^{*}(t)$ of this equation the following holds $x^{*}(0)=q, x^{*}(t) \in M$ for all $t \in(\alpha, \beta)$, where $(\alpha, \beta)$ is the largest definition interval for this solution $(\alpha<0, \beta>0)$.

Theorem 2.1.[Andreev, 2009] Let $x=x(t, \alpha, \varphi)$ be some solution of (1) bounded for all $t \geq \alpha-h_{0}$. Then, 
the set $\omega^{+}(x(t, \alpha, \varphi))$ is quasi-invariant with respect to the family of limiting equations (3).

Let $V: \mathrm{R}^{+} \times \mathrm{C} \rightarrow R$ be a continuous Lyapunov functional candidate. One can define the upper right-hand derivative of $V$ along the solution $x=x(t)$ of (1) as follows

$$
\dot{V}\left(t, x_{t}\right)=\lim _{\Delta t \rightarrow 0+} \frac{1}{\Delta t}\left(V\left(t+\Delta t, x_{t+\Delta t}\right)-V\left(t, x_{t}\right)\right) .
$$

Assume that the upper right-hand derivative of $V$ satisfies the inequality

$$
\dot{V}^{+}\left(t, x_{t}\right) \leq-W(x(t)) \leq 0,
$$

where $W: \mathrm{R}^{p} \rightarrow \mathrm{R}^{+}$is a continuous function.

Theorem 2.2. [Andreev, 2009] Assume that:

1) the solution $x=x(t, \alpha, \varphi)$ of (1) is bounded, i.e. $\left\|x_{t}(\alpha, \varphi)\right\| \leq H \forall t \geq \alpha$;

2) there exists a Lyapunov functional candidate $V=$ $V(t, \varphi)$ such that $V(t, \varphi) \geq m_{1}(H) \forall(t, \varphi) \in \mathrm{R}^{+} \times \mathrm{C}_{H}$, the time derivative $\dot{V}\left(t, x_{t}\right)$ satisfies the inequality (4).

Then $\omega^{+}(x(t, \alpha, \varphi)) \subset M^{*}$, where $M^{*}$ is the maximum quasi-invariant subset of the set $\left\{W^{*}(x)=0\right\}$.

Assume that $f(t, 0) \equiv 0$, so the equation (1) has a zero solution $x(t, \alpha, 0) \equiv 0$.

Introduce the class $\mathcal{K}$ of Hahn-type functions $a$ : $\mathrm{R}^{+} \rightarrow \mathrm{R}^{+}$[Hale, 1977]. The following asymptotic stability theorem holds.

Theorem 2.3. [Andreev, 2009] Assume that:

1) there exists a Lyapunov functional candidate $V=$ $V\left(t, x_{t}\right)$ such that

$$
\begin{array}{r}
a_{1}(|x(t)|) \leq V\left(t, x_{t}\right) \leq a_{2}\left(\left\|x_{t}\right\|\right), \\
\dot{V}\left(t, x_{t}\right) \leq-W(x(t)) \leq 0,
\end{array}
$$

where $a_{1}, a_{2} \in \mathcal{K}$;

2) the set $\{W(x)=0\}$ does not contain the solutions of any limiting equation (3), except for $x=0$.

Then, the zero solution $x=0$ of (1) is uniformly asymptotically stable. If $a_{1}(s) \rightarrow \infty$ as $s \rightarrow \infty$, then, the solution $x=0$ of (1) is uniformly globally asymptotically stable.

Consider the modification of the results presented above in the case when the right-hand side of (1) is a periodic function with respect to a part of the variables.

Let $x=(y, z)^{T}=\left(y_{1}, y_{2}, \ldots, y_{m}, z_{1}, z_{2}, \ldots, z_{s}\right)^{T}$, where $1 \leq m<p, s=p-m$. Hereinafter $(\cdot)^{T}$ is a transposition operation, $y \in \mathrm{R}^{m}$ and $z \in \mathrm{R}^{s}$. The norms of vectors $y \in \mathrm{R}^{m}$ and $z \in \mathrm{R}^{s}$ are denoted by $|y|$ and $|z|$ respectively. Choose the vector norm of $x \in \mathrm{R}^{n}$ in the form $|x|=|y|+|z|$.

Let $C_{(m)}$ and $C_{(s)}$ are spaces of continuous functions $\psi:\left[-h_{0}, 0\right] \rightarrow \mathrm{R}^{m}$ and $\theta:\left[-h_{0}, 0\right] \rightarrow \mathrm{R}^{s}$ according to the norms $\|\psi\|=\max \left(\mid \psi(s),-h_{0} \leq s \leq 0\right)$ and $\left.\|\theta\|=)|\theta(s)|,-h_{0} \leq s \leq 0\right)$. Denote by $\|\varphi\|$ the following norm $\|\varphi\|=\|\psi\|+\|\theta\|$.

Assume that for all $(t, \varphi) \in \mathrm{R}^{+} \times \mathrm{C}$ the following holds $f\left(t, \psi+2 \pi 1_{m}, \theta\right)=f(t, \psi, \theta)$, where $\psi+2 \pi 1_{m}=$ $\left(\psi_{1}+2 \pi, \psi_{2}+2 \pi, \ldots, \psi_{m}+2 \pi\right)^{T}$. Then, the solutions $x=x(t, \alpha, \varphi)$ of (1) can be defined in the space $\mathrm{T}^{m} \times$ $\mathrm{R}^{s}$, where $\mathrm{T}^{m}=\left\{y \in \mathrm{R}^{m}:-\pi \leq y_{j} \leq \pi, j=\right.$ $1,2, \ldots, m\}$.

We introduce the following definition of a positive limit set $\omega^{+}(x(t, \alpha, \varphi))$ of the solution $x=x(t, \alpha, \varphi)$ of (1) in the space $\mathrm{T}^{m} \times \mathrm{R}^{s}$.

Definition 2.4. The point $p=\left(p^{(1)}, p^{(2)}\right) \in \mathrm{T}^{m} \times$ $\mathrm{R}^{s}$ is said to be a limit one for $x=x(t, \alpha, \varphi)$, if there exist the sequences $t_{k} \rightarrow \infty$ and $L^{(k)}=$ $\left(l_{1}^{(k)}, l_{2}^{(k)}, \ldots, l_{m}^{(k)}\right)^{T}, l_{j}^{(k)} \in \mathrm{Z}, j=1,2, \ldots, m$ such that $\left(y\left(t_{k}, \alpha, \varphi\right)-2 \pi L^{(k)}\right) \rightarrow p^{(1)}$ and $z\left(t_{k}, \alpha, \varphi\right) \rightarrow$ $p^{(2)}$ as $k \rightarrow \infty$.

Claim 2.3. Let $x=x(t, \alpha, \varphi)$ be a solution of (1) bounded in $z$, i.e. there exists a positive real $H$ such that $\|z(t, \alpha, \varphi)\| \leq H_{2}=$ const for all $t \geq \alpha$. Then, the set $\omega(x(t, \alpha, \varphi))$ is connected, compact and quasi-invariant with respect to the family of limiting equations (3).

Theorem 2.4. Let one can find Lyapunov functional $V=V(t, \varphi)$ such that:

1) $\exists k \in \mathrm{N}: V\left(t, \psi+2 \pi k 1_{m}, \theta\right)=V(t, \psi, \theta)$; $V(t, \psi, \theta) \geq a(\|\theta\|) \geq m_{1} \forall(t, \psi, \theta) \in \mathrm{R}^{+} \times \mathrm{C}_{(m)} \times$ $\mathrm{C}_{(s)}, a(\|\theta\|) \rightarrow \infty$ as $\|\theta\| \rightarrow \infty$;

2) the upper right-hand time derivative $\dot{V}^{+}\left(t, x_{t}\right)$ along the solutions of (1) satisfies the relationship (4), where the function $W$ is such that $W\left(y+2 \pi 1_{m}, z\right)=W(y, z)$ for all $(y, z) \in \mathrm{T}^{m} \times \mathrm{R}^{s}$.

Then, for a solution $x=x(t, \alpha, \varphi)$ of (1) the following holds $\omega^{+}(x(t, \alpha, \varphi)) \subset M$, where $M$ is the maximally quasi-invariant subset of the set $E=\left\{(y, z) \in \mathrm{T}^{m} \times\right.$ $\left.\mathrm{R}^{s}: W(y, z)=0\right\}$.

\section{Proof.}

Let $x=x(t, \alpha, \varphi)$ be any solution of the system (1). Using the conditions of Theorem one can easily see that $V(t)=V\left(t, x_{t}(\alpha, \varphi)\right) \leq V(\alpha, \varphi)$ and therefore $x(t, \alpha, \varphi)$ is bounded in $z$ for all $t \geq t_{0}$, as well there exists the constant $c_{0}$ such that $V(t, x(t, \alpha, \varphi)) \searrow c_{0}$ as $t \rightarrow+\infty$.

Let $p=\left(p^{(1)}, p^{(2)}\right)^{T} \in \omega^{+}(x(t, \alpha, \varphi))$ be a limit point defined by the sequences $\left\{t_{k}: t_{k} \rightarrow+\infty\right\}$ and $L^{(k)} \in$ Z, i.e. $y^{(k)}=y\left(t_{k}, \alpha, \varphi\right)-2 \pi L^{(k)} \rightarrow p^{(1)}, z^{(k)}=$ $z\left(t_{k}, \alpha, \varphi\right) \rightarrow p^{(2)}$. Note that the functions $x^{(k)}(t)=$ $\left(y\left(t_{k}+t, \alpha, \varphi\right)-2 \pi L^{(k)}, z\left(t_{k}+t, \alpha, \varphi\right)\right)^{T}$ are the solutions of the systems $\dot{x}=f^{(k)}\left(t, x_{t}\right), f^{(k)}(t, \varphi)=$ $f\left(t_{k}+t, \varphi\right)$ in $\mathrm{T}^{m} \times \mathrm{R}^{s}$. Without loss of generality, suppose that $f^{(k)}(t, \varphi) \rightarrow f^{*}(t, \varphi)$ as $k \rightarrow+\infty$. Accordingly $x^{(k)}(t) \rightarrow x^{*}(t)$ uniformly on $t \in[-\mu, \mu]$ $(\mu>0)$ as $k \rightarrow+\infty$ and $x^{*}(t) \equiv f^{*}\left(t, x_{t}^{*}\right)$.

Using the condition 2 of Theorem, for each $t \in[0, \mu]$ 
$(\mu>0)$ one can obtain the following estimate

$$
\begin{array}{r}
V\left(t_{k}+t\right)-V\left(t_{k}-t\right) \leq \\
\leq-\int_{t_{k}-t}^{t_{k}+t} W(x(\tau, \alpha, \varphi)) d \tau= \\
=-\int_{-t}^{t} W\left(x\left(t_{k}+\tau, \alpha, \varphi\right)\right) d \tau= \\
=-\int_{-t}^{t} W\left(x^{(k)}(\tau)\right) d \tau \leq 0 .
\end{array}
$$

Passing to the limit in inequality (5) as $t_{k} \rightarrow+\infty$, one can obtain the following relationship

$$
W\left(x^{*}(t)\right)=0 .
$$

Thus, one can conclude that the set $\omega^{+}(x(t, \alpha, \varphi))$ consists of the solutions $x^{*}(t)$ of limiting system (3) contained in the set $\{W(y, z)=0\}$. This completes the proof.

Let $f(t, 0) \equiv 0$. Then, system (1) has the following set of all equilibrium points

$\left\{\psi(s)=2 \pi k, \theta(s)=0, k=\left(k_{1}, k_{2}, \ldots, k_{p}\right)^{T} \in \mathrm{Z}^{m}\right\}$.

Suppose that on the set of functions $\{\psi(s)=$ $y^{(0)}, \theta(s)=z^{(0)}, y^{(0)} \in \mathrm{T}^{m}, z^{(0)} \in \mathrm{R}^{s},\left\|y^{(0)}\right\|+$ $\left.\left\|z^{(0)}\right\|=\varepsilon>0\right\} \forall t \in \mathrm{R}$ the following inequality holds $\|f(t, y, z)\| \geq \delta(\varepsilon)>0$.

Then, equation (1) as well as the limiting equations (3) cannot have equilibrium positions other than equilibria (7).

Theorem 2.5. Let one can find Lyapunov functional $V=V(t, \varphi)$ such that:

1) $V\left(t, \psi+4 \pi 1_{m}, \theta\right)=V(t, \psi, \theta) \forall(t, \psi, \theta) \in \mathrm{R} \times \mathrm{C}$;

2) $a_{1}(|\varphi(0)|) \leq V(t, \varphi) \leq a_{2}(\|\varphi\|) \forall \varphi \in\{\|\varphi\|<$ $\delta>0\}$

3) $V\left(t, \psi+4 \pi 1_{m}, \theta\right) \geq a_{3}(\|\theta\|) \forall(t, \psi, \theta) \in \mathrm{R}^{+} \times$ $\mathrm{C}_{(m)} \times \mathrm{C}_{(s)}, a_{3}(\|\theta\|) \rightarrow \infty$ as $\|\theta\| \rightarrow \infty$;

4) the upper right-hand derivative of $V$ along the solution of (1) satisfies the inequality (4) where the function $W=W(x)$ is $2 \pi$-periodic in $y$, i.e. $W\left(y+2 \pi 1_{m}, z\right)=$ $W(y, z)$;

5) the set $\{W(x)=0\}$ does not contain solutions of limiting systems (3), except for (7).

Then, the set of equilibrium positions $\left\{x \in \mathrm{R}^{n}: y=\right.$ $\left.4 \pi k, k \in \mathrm{Z}^{m}, z=0\right\}$ of the system (1) is uniformly asymptotically stable. Accordingly, the set of equilibrium positions (7) is globally attractive.

\section{Trajectory Tracking Control Problem of a Serial Robot Manipulator}

Consider the dynamic model of a multi-link robot manipulators defined by the following equations

$$
A(q) \ddot{q}+C(q, \dot{q}) \dot{q}+g(q)+D \dot{q}=u,
$$

where $q \in \mathrm{R}^{n}$ represents the link angles, $A(q) \in \mathrm{R}^{n \times n}$ is the inertia matrix, the Coriolis and centrifugal torques are described by $C(q, \dot{q}) \dot{q}$, the function $g(q)$ represents the gravitational terms, $D \in \mathrm{R}^{n \times n}$ is a constant matrix of the link viscous damping, and $u \in \mathrm{R}^{n}$ is the vector of input torques.

For the robot model (8) define a set $X$ of the reference trajectories as

$$
X=\left\{q^{(0)}(t):[0,+\infty) \rightarrow \mathrm{R}^{n}: \begin{array}{r}
\left|\dot{q}^{(0)}(t)\right| \leq g_{1}, \\
\left.\left|\ddot{q}^{(0)}(t)\right| \leq g_{2}\right\},
\end{array}\right.
$$

where the functions $q^{(0)}(t)$ are differentiable at least two times with respect to $t, g_{i}(i=1,2)$ are some positive constants.

The trajectory tracking control problem can be stated as that of constructing a controller $u=u(t, q, \dot{q})$ which provides a global attractivity for a reference trajectory $q^{(0)}(t) \in X$ of the robot (8).

Let $q^{(0)}(t) \in X$ be some reference trajectory. Define the function $u^{(0)}(t)$ as follows

$$
\begin{array}{r}
u^{(0)}(t)=A\left(q^{(0)}(t)\right) \ddot{q}^{(0)}(t)+ \\
+C\left(q^{(0)}(t), \dot{q}^{(0)}(t)\right) \dot{q}^{(0)}(t)+g\left(q^{(0)}(t)\right)+D \dot{q}^{(0)}(t) .
\end{array}
$$

Define the state errors as

$$
x=q-q^{(0)}(t), \quad \dot{x}=\dot{q}-\dot{q}^{(0)}(t) .
$$

Then, the error dynamic equations are obtained as

$$
\begin{array}{r}
A^{(1)}(t, x) \ddot{x}+C^{(1)}\left(t, x, 2 \dot{q}^{(0)}(t)+\dot{x}\right) \dot{x}+ \\
+R(t, x)+D \dot{x}=u^{(1)}
\end{array}
$$

where

$$
\begin{array}{r}
A^{(1)}(t, x)=A\left(q^{(0)}(t)+x\right), \\
C^{(1)}(t, x, \dot{x})=C\left(q^{(0)}(t)+x, \dot{x}\right), \\
R(t, x)=\left(A\left(q^{(0)}(t)+x\right)-A\left(q^{(0)}(t)\right)\right) \ddot{q}^{(0)}(t)+ \\
+\left(C^{(1)}\left(t, x, \dot{q}^{(0)}(t)\right)-C^{(1)}\left(t, 0, \dot{q}^{(0)}(t)\right)\right) \dot{q}^{(0)}(t)+ \\
+g\left(q^{(0)}(t)+x\right)-g\left(q^{(0)}(t)\right) \\
u^{(1)}=u-u^{(0)}(t) .
\end{array}
$$

In the model of $n$-link robot manipulator with revolute joints the generalized coordinates $q_{1}, q_{2}, \ldots, q_{n}$ denote the link angles. Therefore, the matrices $A^{(1)}(t, x)$, $C^{(1)}\left(t, x, 2 \dot{q}^{(0)}(t)+\dot{x}\right)$ and the vector $R(t, x)$ are periodic functions of $x=\left(x_{1}, x_{2}, \ldots, x_{n}\right)^{T}$ with some pe$\operatorname{riod} h$. Without loss of generality, we may assume that $h=2 \pi$. We will consider the trajectory tracking control problem in the cylindrical phase space

$$
\left\{(x, \dot{x}) \in \mathrm{T}^{n} \times \mathrm{R}^{n}\right\},
$$


where $\mathrm{T}^{n}$ is an $n$-dimensional torus, i.e.

$$
\mathrm{T}^{n}=\left\{x_{1}(\bmod 2 \pi), x_{2}(\bmod 2 \pi), \ldots, x_{n}(\bmod 2 \pi)\right\} .
$$

Assumption 3.1. Assume that the function $R(t, x)$ satisfies the following equality

$$
R(t, x)=F(t, x) p(x) \quad \forall(t, x) \in \mathrm{R}^{+} \times \mathrm{T}^{n},
$$

where $F: \mathrm{R}^{+} \times \mathrm{T}^{n} \rightarrow \mathrm{R}^{n \times n}$ is some bounded continuous function, the vector function $p: \mathrm{T}^{n} \rightarrow \mathrm{R}^{n}$ is bounded continuously differentiable and such that $p(x)=\left(p_{1}\left(x_{1}\right), p_{2}\left(x_{2}\right), \ldots, p_{n}\left(x_{n}\right)\right)^{T}, p(0)=0$ and the following conditions hold

a) $\left|p_{i}\left(x_{i}\right)\right|$ is a periodic function with period $2 \pi \forall x_{i} \in$ $\mathrm{R} ; p_{i}(2 \pi k)=0,\left|p_{i}\left(x_{i}\right)\right|>0 \forall x_{i} \neq 2 \pi k(i=$ $1,2, \ldots, n) \forall k \in \mathrm{Z}$;

b) the function $r(x)=\left(r_{1}\left(x_{1}\right), r_{2}\left(x_{2}\right), \ldots r_{n}\left(x_{n}\right)\right)^{T}$ defined as

$$
\begin{array}{r}
r_{i}\left(x_{i}\right)=\int_{0}^{x_{i}} p_{i}\left(x_{i}\right) d x_{i} \quad \forall x_{i} \in \mathrm{R}, \\
i=1,2, \ldots, n
\end{array}
$$

is bounded continuously differentiable function such that $r_{i}\left(x_{i}\right)$ is a periodic function with period $4 \pi \forall x_{i} \in \mathrm{R}$; $r_{i}(4 \pi k)=0, \quad r_{i}\left(x_{i}\right)>0 \forall x_{i} \neq 4 \pi k \forall k \in \mathrm{Z}, i=$ $1,2, \ldots, n$.

Let us assume that there exists a delay in the manipulator control when measuring the generalized coordinates and when a feedback is functioning. The control action has the form of a nonlinear controller with a delay

$$
u^{(1)}=-B_{1} \dot{x}(t)-B_{2} p\left(x\left(t-h_{1}(t)\right)\right),
$$

where $B_{1}, B_{2} \in \mathrm{R}^{n \times n}$ are diagonal positive definite gain matrices in the control feedback structure, $B_{j}=$ $\operatorname{diag}\left(b_{j 1}, b_{j 2}, \ldots, b_{j n}\right), j=1,2 ; h_{1}(t)$ is a timedependent delay, $0 \leq h_{1}(t) \leq h_{0}=$ const.

Transform the expression of $u^{(1)}$ :

$$
\begin{array}{r}
U^{(1)}=-B_{1} \dot{x}(t)-B_{2} p\left(x\left(t-h_{1}(t)\right)\right)= \\
=-B_{1} \dot{x}(t)-B_{2} p(x(t))+ \\
+\int_{t-h_{1}(t)}^{t} B_{2} \frac{\partial p(x(\tau))}{\partial x} \dot{x}(\tau) d \tau .
\end{array}
$$

By substituting the controller (16) into (12), one can obtain the following closed-loop system

$$
\begin{aligned}
A^{(1)}(t, x) \ddot{x}= & -C^{(1)}\left(t, x, 2 \dot{q}^{(0)}(t)+\dot{x}\right) \dot{x}- \\
-F(t, x) p(x)- & D \dot{x}-B_{1} \dot{x}(t)-B_{2} p(x(t))+ \\
& +\int_{t-h_{1}(t)}^{t} B_{2} \frac{\partial p(x(\tau))}{\partial x} \dot{x}(\tau) d \tau .
\end{aligned}
$$

The limiting systems exist and have a similar form

$$
\begin{array}{r}
A^{(1) *}(t, x) \ddot{x}=-C^{(1) *}\left(t, x, 2 \dot{q}^{(0) *}(t)+\dot{x}\right) \dot{x}- \\
-F^{*}(t, x) p(x)-D \dot{x}-B_{1} \dot{x}(t)-B_{2} p(x(t))+ \\
+\int_{t-h_{1}^{*}(t)}^{t} B_{2} \frac{\partial p(x(\tau))}{\partial x} \dot{x}(\tau) d \tau .
\end{array}
$$

The systems (17) and (18) have the set of all equilibrium points defined as

$$
\Lambda=\left\{(x, \dot{x}) \in \mathrm{T}^{n} \times \mathrm{R}^{n}: \quad p(x)=0, \quad \dot{x}=0\right\} .
$$

One can easily see that the set $\Lambda$ can be written as

$$
\begin{aligned}
& \Lambda=\left\{(x, \dot{x}) \in \mathrm{T}^{n} \times \mathrm{R}^{n}: x=2 \pi k, \dot{x}=0\right. \\
& \left.\forall k=\left(k_{1}, k_{2}, \ldots, k_{n}\right)^{T}, k_{i} \in \mathrm{Z}, i=\overline{1, n}\right\} .
\end{aligned}
$$

Theorem 3.1. Consider the closed-loop system (17). Let Assumption 3.1 hold and let the positive reals $\delta_{i}(i=$ $1,2,3$ ) exist such that the following inequalities hold

$$
\begin{gathered}
\operatorname{lgn} \|-B_{1}+C^{(1)}\left(t, x, S p(x)-\dot{q}^{(0)}(t)\right)-D+ \\
+A^{(1)}(t, x) S \frac{\partial p(x)}{\partial x} \|+\lambda_{0} h_{0} \leq-\delta_{1}, \\
\operatorname{lgn} \|-S B_{2}+S C^{(1)}\left(t, x, \dot{q}^{(0)}(t)\right) S- \\
-S F(t, x) \| \leq-\delta_{2}, \\
\| S\left(C^{(1)}\left(t, x, S p(x)-\dot{q}^{(0)}(t)\right)+\right. \\
+\left(C^{(1)}\left(t, x, \dot{q}^{(0)}(t)\right)\right)^{T}- \\
\left.-D+A^{(1)}(t, x) S \frac{\partial p(x)}{\partial x}\right)-F^{T}(t, x) \| \leq 2 \delta_{3}, \\
\delta_{1} \delta_{2}-\delta_{3}^{2}>0,
\end{gathered}
$$

where $\lambda_{0}=\max \left(\left\|B_{2} \partial p(x) / \partial x\right\|, x \in T^{n}\right), S \in$ $\mathrm{R}^{n \times n}$ is some constant diagonal positive definite matrix, $S=\operatorname{diag}\left(s_{1}, s_{2}, \ldots, s_{n}\right)$.

Then, the set of all equilibrium points of the closedloop system (17) is (20) and it is globally attractive. The subset of (20) defined as

$$
\begin{array}{r}
\Lambda_{1}=\left\{(x, \dot{x}) \in \mathrm{R}^{n} \times \mathrm{R}^{n}: x=4 \pi k, \dot{x}=0\right. \\
\left.\forall k=\left(k_{1}, k_{2}, \ldots, k_{n}\right)^{T}, k_{i} \in \mathrm{Z}, i=\overline{1, n}\right\}
\end{array}
$$

is uniformly asymptotically stable.

\section{Proof.}

Consider the Lyapunov functional candidate $V=$ $V\left(t, x, \dot{x}_{t}\right)$ such as follows

$$
\begin{aligned}
V= & \frac{1}{2}(\dot{x}+S p(x))^{T} A^{(1)}(t, x)(\dot{x}+S p(x))+ \\
& +\Pi(x)+\frac{\lambda_{0}}{2} \int_{-h_{0}}^{0}\left(\int_{\tau}^{0} \dot{x}^{2}(t+s) d s\right) d \tau,
\end{aligned}
$$


where $\Pi=\Pi(x), \Pi: \mathrm{R}^{n} \rightarrow \mathrm{R}^{+}$is a continuously differentiable periodic function with period $4 \pi$ such that $\Pi(x)=\sum_{i=1}^{n} \Pi_{i}\left(x_{i}\right), \Pi_{i}\left(x_{i}\right)=\left(b_{2 i}+s_{i} b_{1 i}\right) \int_{0}^{x_{i}} p_{i}\left(x_{i}\right) d x_{i}$.

It is easy to find out that the functional (24) satisfies conditions 1 and 2 of Theorem 2.5. One can easily see that the following two conditions hold

$$
V\left(t, x, \dot{x}_{t}\right) \equiv 0, t \in \mathrm{R}^{+} \Leftrightarrow(x, \dot{x}) \in \Lambda_{1} .
$$

$$
V\left(t, x, \dot{x}_{t}\right) \rightarrow \infty \text { as }\|\dot{x}\| \rightarrow \infty .
$$

Using Remark (25), one can easily obtain the following

$$
\begin{array}{r}
\dot{V}=\dot{x}^{T}\left(C^{(1)}\left(t, x, S p(x)-\dot{q}^{(0)}(t)\right)-\right. \\
\left.-D-B_{1}+A^{(1)}(t, x) S \frac{\partial p(x)}{\partial x}\right) \dot{x}+ \\
+p^{T}(x)\left(S \left(C^{(1)}\left(t, x, S p(x)-\dot{q}^{(0)}(t)\right)+\right.\right. \\
+\left(C^{(1)}\left(t, x, \dot{q}^{(0)}(t)\right)\right)^{T}- \\
\left.\left.-D+A^{(1)}(t, x) S \frac{\partial p(x)}{\partial x}\right)-F^{T}(t, x)\right) \dot{x}+ \\
+p^{T}(x)\left(S C^{(1)}\left(t, q, \dot{q}^{(0)}(t)\right) S-\right. \\
\left.-F^{T}(t, x) S-B_{2} S\right) p(x)+ \\
+\frac{\lambda_{0}}{2} \int_{t-h_{0}}^{t}\left(\dot{x}^{2}(t)-\dot{x}^{2}(\tau)\right) d \tau .
\end{array}
$$

From (28) using (21) we have the estimate

$$
\begin{array}{r}
\dot{V}\left(t, x, \dot{x}_{t}\right) \leq-\delta_{1}\|\dot{x}\|^{2}+ \\
+2 \delta_{3}\|\dot{x}\|\|p(x)\|-\delta_{2}\|p(x)\|^{2}- \\
-\frac{\lambda_{0}}{2} \int_{t-h_{0}}^{t}(\dot{x}(t)-\dot{x}(\tau))^{2} d \tau \leq \\
\leq-\varepsilon_{0}\left(\|\dot{x}\|^{2}+\|p(x)\|^{2}\right) \leq 0,
\end{array}
$$

where $\varepsilon_{0}>0$ is some constant.

From (29) one can easily see that $\dot{V}\left(t, x, \dot{x}_{t}\right)$ is a negative semi-definite functional.

Note that $\left\{\dot{V}^{*}=0\right\} \subset\{\dot{x}=0, p(x)=0\}$. This set can only contain equilibrium positions (20) of the limiting systems (18). Based on Theorem 2.5, one can obtain the proof of Theorem.

Let the control action have the form of a nonlinear controller with a delay

$$
u^{(1)}=-B_{1} \dot{x}\left(t-h_{1}(t)\right)-B_{2} p\left(x\left(t-h_{2}(t)\right)\right),
$$

where $B_{1}, B_{2} \in \mathrm{R}^{n \times n}$ are diagonal positive definite gain matrices in the control feedback structure, $B_{j}=$ $\operatorname{diag}\left(b_{j 1}, b_{j 2}, \ldots, b_{j n}\right), j=1,2 ; h_{1}(t), h_{2}(t)$ are timedependent delays, $0 \leq h_{i}(t) \leq h_{0}=$ const, $i=1,2$.

By substituting the controller (30) into (12), one can obtain the following closed-loop system

$$
\begin{array}{r}
A^{(1)}(t, x) \ddot{x}=-C^{(1)}\left(t, x, 2 \dot{q}^{(0)}(t)+\right. \\
+\dot{x}) \dot{x}-F(t, x) p(x)-D \dot{x}- \\
-B_{1} \dot{x}\left(t-h_{1}(t)\right)-B_{2} p\left(x\left(t-h_{2}(t)\right)\right) .
\end{array}
$$

Transform the expression of $u^{(1)}$ :

$$
\begin{array}{r}
u^{(1)}=-B_{1} \dot{x}\left(t-h_{1}(t)\right)-B_{2} p\left(x\left(t-h_{2}(t)\right)\right)= \\
=-B_{1} \dot{x}(t)+\int_{t-h_{1}(t)}^{t} B_{1} \ddot{x}(\tau) d \tau-B_{2} p(x(t))+ \\
+\int_{t-h_{2}(t)}^{t} B_{2} \frac{\partial p(x(\tau))}{\partial x} \dot{x}(\tau) d \tau= \\
=-B_{1} \dot{x}(t)-B_{2} p(x(t))- \\
-\int_{t-h_{1}(t)}^{t} B_{1}\left(A^{(1)}(\tau, x(\tau))\right)^{-1} \times \\
\times\left(C^{(1)}\left(\tau, x(\tau), 2 \dot{q}^{(0)}(\tau)+\dot{x}(\tau)\right) \dot{x}(\tau)+\right. \\
+F(\tau, x(\tau)) p(x(\tau))+D \dot{x}(\tau)+ \\
B_{1} \dot{x}\left(\tau-h_{1}(\tau)\right)+B_{2} p\left(x\left(\tau-h_{2}(\tau)\right)\right) d \tau+ \\
+\int_{t-h_{2}(t)}^{t} B_{2} \frac{\partial p(x(\tau))}{\partial x} \dot{x}(\tau) d \tau .
\end{array}
$$

Theorem 3.2. Consider the closed-loop system (31). Let Assumption 2.1 hold and let positive reals $\delta_{i}(i=$ $1,2,3$ ) exist such that the following inequalities hold

$$
\begin{array}{r}
\operatorname{lgn} \|-B_{1}+C^{(1)}\left(t, x, S p(x)-\dot{q}^{(0)}(t)\right)-D+ \\
+A^{(1)}(t, x) S \frac{\partial p(x)}{\partial x} \|+4 h_{0} \lambda_{1} \leq-\delta_{1}, \\
\operatorname{lgn} \|-S B_{2}+S C^{(1)}\left(t, x, \dot{q}^{(0)}(t)\right) S- \\
-S F(t, x) \|+4 h_{0} \lambda_{1} \leq-\delta_{2} \\
\| S\left(C^{(1)}\left(t, x, S p(x)-\dot{q}^{(0)}(t)\right)+\right. \\
+\left(C^{(1)}\left(t, x, \dot{q}^{(0)}(t)\right)\right)^{T}- \\
\left.-D+A^{(1)}(t, x) S \frac{\partial p(x)}{\partial x}\right)-F^{T}(t, x) \| \leq 2 \delta_{3}, \\
\delta_{1} \delta_{2}-\delta_{3}^{2}>0,
\end{array}
$$




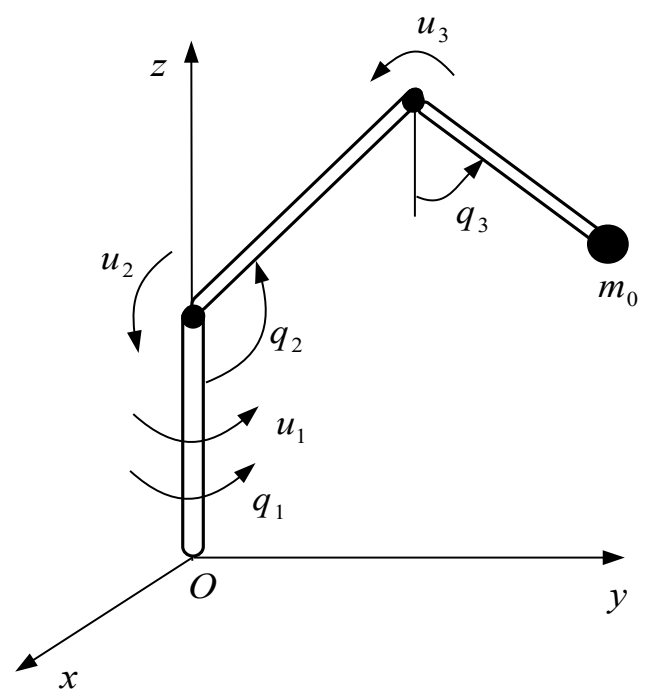

Figure 1. Model of a 3-DOF robot manipulator.

where $S \in \mathrm{R}^{n \times n}$ is some constant diagonal positive definite matrix, $S=\operatorname{diag}\left(s_{1}, s_{2}, \ldots, s_{n}\right)$ and

$$
\begin{array}{r}
\lambda_{1}=\sup \left(\| B_{1}\left(A^{(1)}(t, x)\right)^{-1} \times\right. \\
\times\left(C^{(1)}\left(t, x, 2 \dot{q}^{(0)}(t)+\dot{x}\right)+D\right) \|+ \\
\left\|B_{1} \frac{\partial p(x)}{\partial x}\right\|+\left\|B_{1}\left(A^{(1)}(t, x)\right)^{-1} B_{1}\right\|, \\
\left\|B_{1}\left(A^{(1)}(t, x)\right)^{-1} F(t, x)\right\|+ \\
+\left\|B_{1}\left(A^{(1)}(t, x)\right)^{-1} B_{2}\right\|, \\
\left\|S B_{1}\left(A^{(1)}(t, x)\right)^{-1}\left(C^{(1)}\left(t, x, 2 \dot{q}^{(0)}(t)+\dot{x}\right)+D\right)\right\|+ \\
+\left\|S B_{1}\left(A^{(1)}(t, x)\right)^{-1} B_{1}\right\|, \\
\left\|S B_{1}\left(A^{(1)}(t, x)\right)^{-1} F(t, x)\right\|+ \\
+\left\|S B_{1}\left(A^{(1)}(t, x)\right)^{-1} B_{2}\right\| \forall x \in \mathrm{T}^{n}, \\
\|\dot{x}\| \leq \gamma=\text { const }) .
\end{array}
$$

Then, the zero solution $x=\dot{x}=0$ of the closed-loop system (17) is semi-globally uniformly asymptotically stable.

\section{Proof.}

Consider the Lyapunov functional candidate $V=$ $V\left(t, x_{t}, \dot{x}_{t}\right)$ such as follows

$$
\begin{aligned}
V= & \frac{1}{2}(\dot{x}+S p(x))^{T} A^{(1)}(t, x)(\dot{x}+S p(x))+\Pi(x)+ \\
& +\lambda_{1} \int_{-2 h_{0}}^{0}\left(\int_{\tau}^{0}\left(\dot{x}^{2}(t+s)+p^{2}(x(t+s))\right) d s\right) d \tau,
\end{aligned}
$$

where $\Pi=\Pi(x)$ is defined by (25).

Note that $V\left(t, x, \dot{x}_{t}\right)$ is a positive semi-definite functional.
One can easily obtain the following estimate

$$
\begin{array}{r}
\dot{V} \leq-\delta_{1}\|\dot{x}(t)\|^{2}+ \\
+2 \delta_{3}\|\dot{x}(t)\|\|p(x(t))\|-\delta_{2}\|p(x(t))\|^{2}+ \\
+\|(\dot{x}(t)+S p(x(t)))^{T} \times \\
\times\left(-B_{1} \dot{x}\left(t-h_{1}(t)\right)-B_{2} p\left(x\left(t-h_{2}(t)\right)\right)\right) \|- \\
-2 \lambda_{1} h_{0}\left(\dot{x}^{2}(t)+\|p(x(t))\|^{2}\right)- \\
-\int_{t-2 h_{0}}^{t}\left(\|\dot{x}(\tau)\|^{2}+\|p(x(\tau))\|^{2}\right) d \tau \leq \\
\leq-\varepsilon_{0}\left(\|\dot{x}(t)\|^{2}+\|p(x(t))\|^{2}\right)- \\
-\lambda_{1} \quad \int_{t-2 h_{0}}^{t}\left(\|\dot{x}(t)\|^{2}+\|p(x(t))\|^{2}+\right. \\
+\left\|x_{1}(\tau)\right\|^{2}+\|p(x(\tau))\|^{2}-\|\dot{x}(t)\|\|\dot{x}(\tau)\|- \\
-\|\dot{x}(t)\|\|p(x(\tau))\|-\|p(x(t))\| \| \dot{x}(\tau)) \|- \\
-\|p(x(t))\|\|p(x(\tau))\|) d \tau \leq \\
\leq-\varepsilon_{0}\left(\|\dot{x}(t)\|^{2}+\|p(x(t))\|^{2}\right) \leq 0,
\end{array}
$$

where $\varepsilon_{0}>0$ is some constant.

From (35) one can easily see that $\dot{V}\left(t, x_{t}, \dot{x}_{t}\right)$ is a negative semi-definite functional. Further, similarly to the proof of Theorem 3.1, one can obtain the required result.

Remark 3.1. The stabilizability conditions (33) for $h_{0}=0$ coincide with ones obtained in [Hale, 1977] without taking into account the delay in the feedback structure. Thus, the addition of the inequalities included in (33) by the terms $\lambda_{0} h_{0}$ and $4 \lambda_{1} h_{0}$ allows us to estimate the admissible value of the delay in control solving the problem of global and semi-global trajectory tracking control of the robot manipulator with cylindrical joints.

\section{Trajectory tracking of a 3-DOF robotic manipu- lator}

In this section, the performance of the proposed controller for the 3-DOF robotic manipulator (see, Fig. 1) is illustrated. Assume that the generalized coordinates $q_{1}=\varphi_{1}, q_{2}=\varphi_{2}$, and $q_{3}=\varphi_{3}$ are the angular displacements of the revolute joints $O_{1}, O_{2}$, and $O_{3}$ respectively. The dynamics of a 3-DOF serial robot manipulator with revolute joints is defined by (8).

The elements $d_{i j}$ of the matrix $A(q)$ are as follows:

$$
\begin{array}{r}
a_{11}=J_{01}+m_{2} r_{2}^{2} \sin ^{2}\left(q_{2}\right)+ \\
+m_{30}\left(l_{2} \sin \left(q_{2}\right)+r_{3} \sin \left(q_{3}\right)\right)^{2}, \\
a_{12}=a_{13}=a_{21}=a_{31}=0, \\
a_{22}=m_{2} r_{2}^{2}+m_{3} l_{2}^{2} \\
a_{23}=a_{32}=m_{30} l_{2} r_{3} \cos \left(q_{2}-q_{3}\right) / 2, \\
a_{33}=m_{30} r_{3}^{2} .
\end{array}
$$

The elements $c_{i j}$ of the matrix $C(q, \dot{q})$ are as follows:

$$
c_{11}=\left(m_{2} r_{2}^{2}+m_{30} l_{2}^{2}\right) \sin \left(2 q_{2}\right) \dot{q}_{2} / 2
$$

$+m_{30} l_{2} r_{3}\left(\sin \left(q_{2}\right) \cos \left(q_{3}\right) \dot{q}_{3}+\cos \left(q_{2}\right) \sin \left(q_{3}\right) \dot{q}_{2}\right)$ 


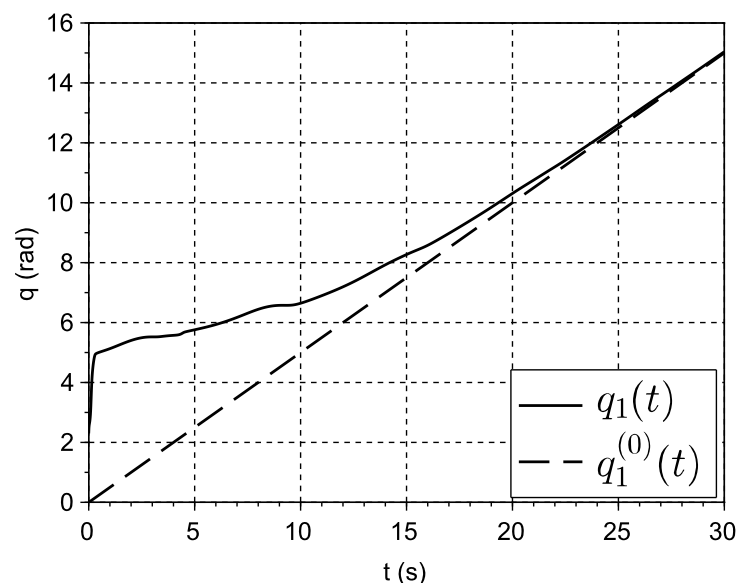

Figure 2. The time response of angular position and reference of the manipulator first link.

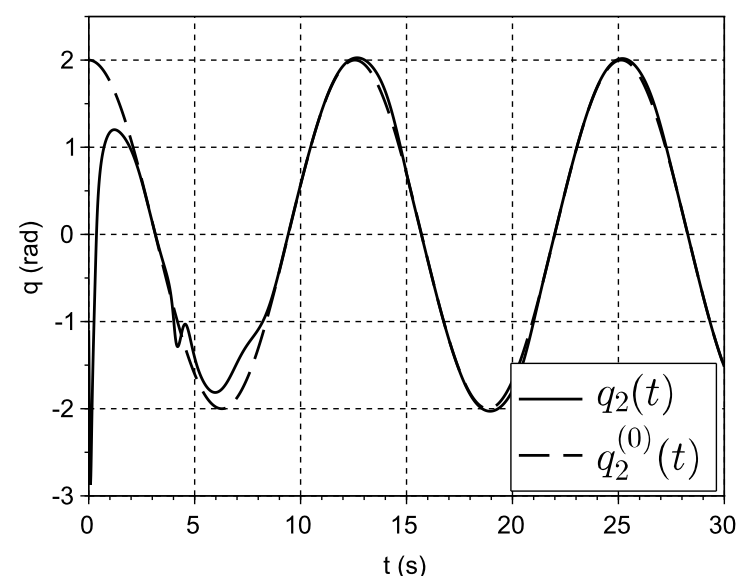

Figure 3. The time response of angular position and reference of the manipulator second link.

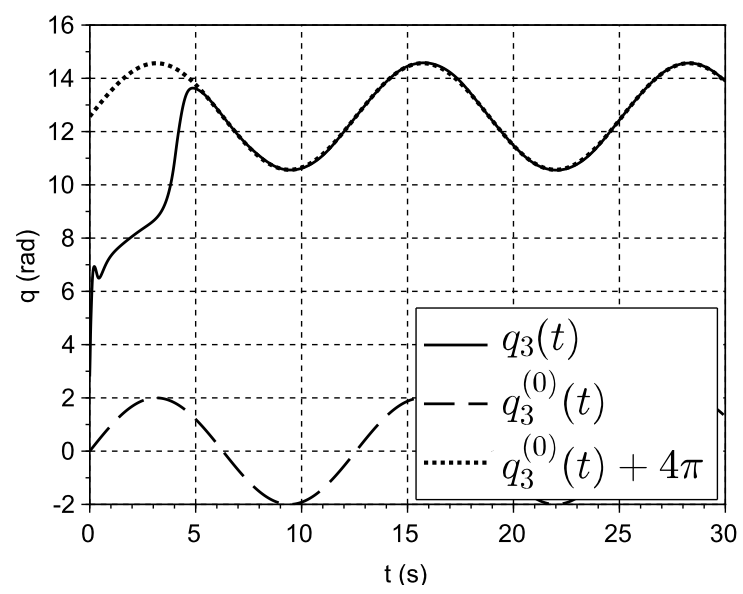

Figure 4. The time response of angular position and reference of the manipulator third link.

$$
\begin{array}{r}
c_{12}=-c_{21}=\left(m_{2} r_{2}^{2}+m_{30} l_{2}^{2}\right) \sin \left(2 q_{2}\right) \dot{q}_{1} / 2 \\
+m_{30} l_{2} r_{3} \sin \left(q_{3}\right) \cos \left(q_{2}\right) \dot{q}_{1}, \\
c_{13}=-c_{31}=m_{30} l_{2} r_{3} \sin \left(q_{2}\right) \cos \left(q_{3}\right) \dot{q}_{1} \\
+m_{30} r_{3}^{2} \sin \left(2 q_{3}\right) \dot{q}_{1} / 2, c_{22}=c_{33}=0, \\
c_{23}=m_{30} l_{2} r_{3} \sin \left(q_{2}-q_{3}\right) \dot{q}_{3} / 2, \\
c_{32}=-m_{30} l_{2} r_{3} \sin \left(q_{2}-q_{3}\right) \dot{q}_{2} / 2 .
\end{array}
$$

The elements $g_{i}$ of the vector $g(q)$ are as:

$$
\begin{array}{r}
g_{1}=0, g_{2}=\left(m_{2} r_{2}+m_{30} l_{2}\right) g \sin q_{2}, \\
g_{3}=m_{30} r_{3} g \sin q_{3} .
\end{array}
$$

The robot parameters are given as

$$
\begin{array}{r}
J_{01}=0,09 \mathrm{~kg} \cdot \mathrm{m}^{2}, \\
m_{2}=3 \mathrm{~kg}, \quad m_{3}=5 \mathrm{~kg}, \quad m_{0}=3 \mathrm{~kg}, \\
l_{2}=0,5 \mathrm{~m}, \quad r_{2}=0,2 \mathrm{~m}, \quad r_{3}=0,47 \mathrm{~m} .
\end{array}
$$

The desired trajectory is chosen as

$$
\begin{aligned}
q_{1}^{(0)}(t)=0,5 t \mathrm{rad}, & q_{2}^{(0)}(t)=2 \cos (0,5 t) \mathrm{rad} \\
q_{3}^{(0)}(t) & =2 \sin (0,5 t) \mathrm{rad} .
\end{aligned}
$$

The controller is given by (15), where

$$
p(x)=\left(\sin \left(x_{1} / 2\right), \sin \left(x_{2} / 2\right), \sin \left(x_{3} / 2\right)\right)^{T} .
$$

Let $h=0.1 \mathrm{~s}$. The control gain parameters are chosen such as $b_{1}=-10, \quad b_{2}=-3.5$.

Consider the simulations results using the initial conditions for the robot manipulator such as

$$
\begin{array}{r}
q_{1}(0)=2,3+q_{1}^{(0)}(0) \mathrm{rad}, \\
q_{2}(0)=-3,1+q_{2}^{(0)}(0) \mathrm{rad}, \\
q_{3}(0)=2,8+q_{3}^{(0)}(0) \mathrm{rad}, \\
\dot{q}_{1}(0)=30 \mathrm{rad} / \mathrm{s}, \dot{q}_{2}(0)=-20 \mathrm{rad} / \mathrm{s}, \\
\dot{q}_{3}(0)=35 \mathrm{rad} / \mathrm{s} .
\end{array}
$$

In Figures $2-4$ we show the link trajectories as well as the references for the robot (8). From these results, it can be seen that controller (15) provides asymptotic convergence to the reference trajectory plus $2 \pi k$, where $k=\left(k_{1}, k_{2}, k_{3}\right)^{T}, k_{i} \in \mathrm{Z}, i=1,2,3$.

\section{Conclusion}

In this paper, in a nonlinear formulation, the problems of global and semi-global trajectory tracking control of a multi-link manipulator with cylindrical joints are solved. The constructed control scheme consists of a nonlinear 
feedback term taking into account the delay of the input signals and a compensator of inertial torques and computed torques along the tracked trajectory. Thus, the compensator is partial since there is no need to compensate for the above-mentioned torques on the disturbed motion. Moreover, the positive effect of the torques of external forces on the stabilization process can be used. In contrast to the controller based on the predictor approach, the input signals can have delays expressed by rather arbitrary bounded time functions. Estimates of the maximum delay are a priori determined theoretically depending on the parameters of the tracked trajectory and the mass-inertial parameters of the system. The use of a cylindrical phase space allows one to take into account the periodicity of angular coordinates and, thereby, the physical nature of trajectory tracking. Thus, a decrease in energy consumption for the stabilization process is achieved. The control structure is demonstrated in solving the semi-global stabilization problem of a three-link manipulator with cylindrical joints. Such a model of manipulator makes it possible to clearly visualize the dynamic properties of the controlled motion.

\section{References}

Aguinaga-Ruiz, E., Zavala-Rio, A., Santibánez, V., and Reyes, F. (2009). Global trajectory tracking through static feedback for robot manipulators with bounded inputs. IEEE Transactions on Control Systems Technology, 17 (4), pp. 934-944.

Andreev, A. (1984). On the asymptotic stability and instability of the zeroth solution of a non-autonomous system. Journal of Applied Mathematics and Mechanics, 48 (2), pp. 154-160.

Andreev, A.S. (2009). The Lyapunov functionals method in stability problems for functional differential equations. Automation and Remote Control, 70, pp. 14381486.

Andreev, A.S., and Peregudova, O.A. (2020). On global trajectory tracking control of robot manipulators in cylindrical phase space. International Journal of Control, 93 (12), pp. 3003-3015.

Bagheri, M., Naseradinmousavi, P., and Krstic, M. (2019). Feedback linearization based predictor for time delay control of a high-DOF robot manipulator. Automatica, 108, 108485.

Bekiaris-Liberis, N. and Krstic, M. (2017). Predictorfeedback stabilization of multi-input nonlinear sys- tems. IEEE Transactions on Automatic Control, 62 (2), pp. 516-531.

Castaneda, L.A., Luviano-Juareza, A., Ochoa-Ortega, G., and Chairez, I. (2018). Tracking control of uncertain time delay systems: An ADRC approach. Control Engineering Practice, 78, pp. 97-104.

Fischer, N., Dani, A., Sharma, N., and Dixon, W.E. (2013). Saturated control of an uncertain nonlinear system with input delay. Automatica, 49 (6), pp. 17411747.

Hale, J.K. (1977). Theory of Functional Differential Equations. Springer-Verlag, New York.

Khalil, H. (2001). Nonlinear systems. Pearson.

Kim, J.H., Hur, S.M., and Oh, Y. (2017). Performance analysis for bounded persistent disturbances in PD/PID-controlled robotic systems with its experimental demonstrations. International Journal of Control, DOI:10.1080/00207179.2017.1288301.

Moreno-Valenzuela, J., Santibánez, V., OrozcoManriquez, E., and González-Hernández, L. (2010). Theory and experiments of global adaptive output feedback tracking control of manipulators. IET Control Theory and Applications, 4 (9), pp. 1639-1654.

Nunes, E. V. L., Hsu, L., and Lizarralde, F. (2008). Arbitrarily small damping allows global output feedback tracking of a class of Euler-Lagrange systems. American Control Conference, Westin Seattle Hotel, Seattle, Washington, USA, pp. 377-382.

Oliveira, T.R., Peixoto, A.J., and Hsu, L. (2015). Global tracling for a class of uncertain nonlinear systems with unknown sign-switching control direction by output feedback. International Journal of Control, 88(9), pp. 1895-1910.

Penaloza-Mejia O., Alvarez J., and Marques-Martinez, L.A. (2013). Discontinuous control design for tracking feasible trajectories in underactuated maniulators. Cybernetics and Physics, 2 (3), pp. 143-150.

Rigatos, G.G. (2020). Differential flatness theory-based control and filtering for a mobile maniulator. Cybernetics and Physics, 9 (1), pp. 57-68.

Spong, M., Seth, H., and Vidyasagar, M. (2004). Robot Dynamics and Control. Wiley, New York.

Utkin, V., Poznyak, A., Orlov, Y., and Polyakov, A. (2020). Conventional and high order sliding mode control. Journal of the Franklin Institute, 357 (15), pp. 10244-10261. 